\title{
MEASURE CHARACTERIZATIONS AND PROPERTIES OF NORMAL AND REGULAR LATTICES
}

\author{
PETER M. GRASSI \\ Department of Mathematics \\ Hofstra University \\ Hempstead, New York 1155,0 \\ (Recelved October 15, 1989)
}

ABSTRACT. Various equivalent characterizations of normaltey are considered and a measure theoretic definition is given for strongly normal lattices. Measure conditions related to the space of o-smooth, lattlce-regular, 0-1 measures are noted which imply, or are equivalent to, the space being Hausdorff, regular, or prime complete.

KEY WORDS AND PHRASES. Lattice, zero-one measures, sigma-smooth, normal, regular, Hausdorff, prime complete, strongly normal, filter, disjunctive. 1980 AMS SUBJECT CLASSIFICATION CODE. 28A60, 28A32.

\section{INTRODUCTION AND BACKGROUND.}

Our concerns in this paper are two-fold. First, we wish to consider in detail and from a measure theoretic point of view, characterizations of normal and strongly normal lattices. This is desirable since tt is more natural when pairs of lattices are considered, one of which is contalned in the other, to consider measure extensions and restrictions. Also, many results hold equally well for a more general measure than $0-1$ valued measures.

Second, we initiate in some detall a study of reflections of lattice properties to the Wallman replete space $I_{R}{ }^{\sigma}(L)$ (see below for definitions), and conversely how properties of this space reflect back to the underlying lattice. This space, except in special topological cases, has not been throughly investigated and is not as wellunderstood as the compact Wallman space $I_{R}(L)$.

We begin with a brief review of the relevant Lattice definitions, and the related topological spaces involved. Our notation and terminology is consistent with those in the 11terature (see e.g. Grass1 [1], Huerta [2], Nöbeling [3], and Szeto [4,5]. Further detalls concerning $I_{R}{ }^{\sigma}(L)$ can be found in (Grassi [1,7] and Fro11k [6], [4]) but these will not be necessary for reading this paper.

\section{NOTATION AND TERMINOLOGY.}

We sha1l let $L$ denote a lattlce of subsets of a set $X$ and shall assume that the empty set and $X$ are in L. (L) and $\sigma(L)$ denote the algebra and $\sigma-a 1 g e b r a$ respectively generated by $L$. If $L$ is closed under countable intersections then $L$ is said to be 
a $\delta$-lattice. $\quad \tau$ will denote the lattice consisting of arbitrary intersections of elements of $L$. $L$ is sald to be normal if whenever $A, B \in L$ such that $A \cap B=\phi$, there exist $C, D \in L$ such that $A \& C^{\prime}, B<D^{\prime}$ and $C^{\prime} / D^{\prime}=\phi$. Equivalently, $L$ is normal if for all $L \in L$, if $L \subset L_{1}$ ' $U L_{2}$ ' where $L_{1}$ and $L_{2} \in L$ then there exist $A_{1}, A_{2} \varepsilon L$ such that $L=A_{1} / A_{2}$ and $A_{1} C_{-} L_{1}^{\prime}, A_{2} C L_{2}^{\prime} \cdot$ is regular if for each $x \in X$ and $A \in L$ such that $X \& A$, there exist $B, C \in L$ with $x \in B^{\prime}, A^{\prime}=C^{\prime}$ and $B^{\prime} r_{i} C^{\prime}=\phi . \quad$ Similar definitions apply to $L$ being separating and $I_{2}$, which are analogous to the topological definitions, replacing closed sets with lattice sets. L is complement generated if for al1 L $\varepsilon L, L=\bigcap A_{n}^{\prime}, A_{n} \varepsilon L$. $L$ is disjunctive if for al1 $x \in X \quad$ and $A \in L$ with $X=\$ A$, there exists $B \in L$ such that $x \in B$ and $A \cap B=\Phi$. $L$ is countably paracompact if whenever $\left\{A_{n}\right\}$ is a decreasing sequence of lattice sets in which $\bigcap_{n=1}^{+\infty} A_{n}=\phi$, there exists a decreasing sequence of $L^{\prime}$ sets $\left\{B_{n}^{\prime}\right\}$ such that $A_{n} C B_{n}^{\prime}$ for all $n$ and $\bigcap_{n=1}^{+\infty} B_{n}^{\prime}=\phi$.

If $L_{1}$ and $L_{2}$ are lattices of subsets of $X$ and $L_{1} \subset L_{2}$ then $\underline{L}_{1}$ separates $\underline{L}_{2}$ if whenever $A, B \in L_{2}$ such that $A \cap B=\phi$, there exist $C, D \in L_{1}$ such that $A C C$, BCD and $C \cap D=\phi ; L_{1}$ semiseparates $L_{2}$ if whenever $A \varepsilon L_{1}$ and $B \varepsilon L_{2}$ such that $A \cap B=\phi$, there exists $C \in L_{1}$ such that $B C . C$ and $A \cap C=\phi$. If $L_{1}$ separates $L_{2}$ then $L_{1}$ is normal if and only if $\mathrm{L}_{2}$ is normal.

$\Pi(L)$ will denote the set of all premeasures on $L$, and $I(L)$ the set of all $0-1$ finitely additive measures defined on $A(L) . \quad I_{R}(L)$ will denote the subset of $I(L)$ consisting of al1 $0-1$ L-regular measures and $I_{R}{ }^{\sigma}$ (L) that subset of $I_{R}(L)$ consisting of $\sigma$-smooth, 0-1, L-regular measures. $I_{\sigma}(L)$ denotes those measures in $I(L)$ which are o-smooth on L. $M(L)$ denotes the set of all finitely additive measures defined on $A(L)$. Without 1088 of generality, we assume that these measures are non-negative. We note that there is a one-to-one correspondence between filters on $L$ and premeasures on $L$, between prime filters on $L$ and measures In $I(L)$, and between L-ultrafilters and measures in $I_{R}(L)$. Furthermore, a prime filter on $L$ has the countable intersection property if and only' if the corresponding measure is in $I_{\sigma}(L)$. If $\mu \varepsilon M(L), S(\mu)$ denotes the support of $\mu$. $L$ is said to be replete (prime complete) if $S(\mu) \neq \phi$ for all $\mu \in I_{R}^{\sigma}\left(I_{\phi}\right)$ ( $\mu \in I_{\sigma}(L t)$. If $\mu, v \in M(L)$ (or $\Pi(L)$ we will write $\mu<v(L)$ whenever $\mu(L)<v(L)$ for al1 L $\varepsilon$ L. It 18 we11-known that if $\mu \in I(L)$ then there exists a $v \varepsilon I_{R}(L)$ such that $\mu<v(L)$. Also, $L$ is $T_{2}$ if and only if for every $\mu \varepsilon I(L), S(\mu)=\phi$ or a singleton; L is regular if and only if whenever $\mu<v(L), \mu, v \varepsilon I(L), S(\mu)=S(v)$.

A mapping $T: X+R$, where $R$ is the real numbers with the usual topology, is L-continuous if $\mathrm{T}^{-1}$ (C) $\varepsilon \mathrm{L}$ for all closed sets $\mathrm{C}$ of $\mathrm{R}$. $\mathrm{Z}(\mathrm{L})$ denotes the lattice of all zero-sets of $\mathrm{L}$-continuous functions (1.e. $\mathrm{Z} \varepsilon \mathrm{Z}(\mathrm{L}$ ) If and only if there exists an L-continuous function $T$ such that $\left.Z=T^{-1}(\{0\})\right)$. If $X$ is a topological space, $F$ will denote the closed sets, $O$ the open sets, and $Z$ the zero sets of $X$. When discussing the zero sets of a topological space $X$, we will always assume, without explicit mention, that $X$ is completely regular. 
For $L \in \mathbf{L}, W(L)=\left(\mu \varepsilon I_{R}(L) \mid \mu(L)=1\right\}$, and $W_{\sigma}(L)=\left\{\mu \varepsilon I_{R}^{\sigma}(L) \mid \mu(L)=1\right\}$. $W(\mathbf{L})=\{W(L) \mid L \varepsilon L)$, and $W_{\sigma}(\mathbf{L})=\left\{W_{\sigma}(L) \mid L E \mathbf{L}\right\}$. The space $I_{R}(\mathbf{L})$, with $T W(L)$ as the closed sets (i.e. the Wallman topology) is a compact, $T_{1}$ topological space. If $L$ is disjunctive then $I_{R}(L)$ is $T_{2}$ if and only if $L$ is normal. Also, if $L$ is disjunctive then $I_{R}{ }^{\sigma}(L)$ is $W_{\sigma}(L)$-replete and is $W_{\sigma}(L)$-disjunctive.

3. ON NORMAL LATTICES.

In this section we consider a variety of new characterizations of normal lattices from a measure theoretic point of view, as well as consequences of a lattice being normal. Also, the concept of a strongly normal lattice is introduced.

We begin with the following equivalent characterizations of normality. A. Koltun (unpublished) proved that statements 2 and 3 below are equivalent. We will essentially use his proof to show that 2 implies 3 .

THEOREM 3.1. Let $v \in I_{R}(\mathbf{L})$ and $\rho \in I_{R}\left(L^{\prime}\right)$ where $v<\rho\left(L^{\prime}\right)$. For any $E(X$ define $v^{\prime}(E)=\inf v\left(L^{\prime}\right)$ and $\rho^{\prime}(E)=\inf \rho\left(L^{\prime}\right)$ where $E \subset L^{\prime}, L \varepsilon$ L. The following are then equivalent:

1. $\mathbf{v}^{\prime}=\rho^{\prime}$ on $\mathbf{L}$.

2. For each $\mu \in I(\mathbf{L})$, there exists a unique $\mu_{1} \varepsilon I_{R}(\mathbf{L})$ such that $\mu<\mu_{1}(\mathbf{L})$.

3. L is normal.

PRoof. ( 1 implies 2.) Suppose $v^{\prime}=\rho^{\prime}$ on L. Let $\mu<\mu_{1}(\mathbf{L})$ and $\mu<\mu_{2}(\mathbf{L})$ where $\mu \varepsilon I(L)$ and $\mu_{1}, \mu_{2} \varepsilon I_{R}^{(L)}$. Then there exists a $\rho \varepsilon I_{R}\left(L^{\prime}\right)$ such that

$\mu<\rho\left(\mathbf{L}^{\prime}\right)$. Therefore $\rho<\mu<\mu_{1}(\mathbf{L})$ and $\rho<\mu<\mu_{2}(\mathbf{L})$. Therefore $\mu_{1}=\mu_{2}$ on $\mathbf{L}$ and hence $\mu_{1}=\mu_{2}$ ( (2 implies 3.) Suppose $L$ is not normal. Then there exists $A_{1}, A_{2} \varepsilon L$ such that $A_{1} \cap A_{2}=\phi$ and $B=\left\{B^{\prime} \varepsilon L^{\prime} \mid A_{1} \subset B^{\prime}\right.$ or $\left.A_{2} \subset B^{\prime}\right\}$ has the finite intersection property. Therefore, there exists $\mu \varepsilon I(L)$ such that $\mu\left(B^{\prime}\right)=1$ for all $B^{\prime} \varepsilon$ H. If $B \varepsilon \mathbf{L}$ such that $\mu(B)=1$ then it follows that $A_{1} \cap B \neq \phi$ and $A_{2} \cap B \neq \phi$. Therefore, there exist $\mu_{1}, \mu_{2} \in I_{R}(L)$ such that $\mu_{1}\left(A_{1}\right)=1, \mu<\mu_{1}(\mathbf{L})$ and $\mu_{2}\left(A_{2}\right)=1, \mu<\mu_{2}(L)$. Clearly $\mu_{1} \neq \mu_{2}$. (3 implies 1.) . Suppose $v^{\prime}(A)=v(A)=0$, A $\varepsilon$ L. Then $v\left(A^{\prime}\right)=1$. Since $v \varepsilon I_{R}(L)$, there exists $L \subset A^{\prime}$, $L \varepsilon L$, such that $v(L)=1$. Since $L$ is normal, there exist $C, D \varepsilon L$ such that $A C$ $C^{\prime} C . D<L^{\prime}$. Therefore, $\rho\left(C^{\prime}\right)<\rho(D)<v(D)=0$ and hence $\rho^{\prime}(A)=0$.

We will now define what appears to be a condition weaker than $L_{1}$ separates $L_{2}$, but we shall show they are equivalent.

DEFINITION 3.1. $\underline{L}_{1}$ partiy separates $\underline{L}_{2}$ if whenever A,B $\varepsilon L_{2}$ and $A C^{-} B^{\prime}$, there exists $C \in L_{1}$ such that $A-C C_{-} B^{\prime}$.

LEMMA 3.1. $\mathrm{L}_{1}$ part1y separates $\mathrm{L}_{2}$ if and on1y if $\mathrm{L}_{1}$ separates $\mathrm{L}_{2}$. PROOF. Clearly, if $\mathbf{L}_{1}$ separates $L_{2}$ then $L_{1}$ partly separates $L_{2}$. Suppose $L_{1}$ partiy separates $L_{2}{ }^{\circ} \quad$ Then $L_{1}$ semiseparates $L_{2}{ }^{*}$ Therefore the restriction map $\Psi: I_{R}\left(L_{2}\right)+I_{R}\left(L_{1}\right)$ is well defined. If $L_{1}$ does not separate $L_{2}$ then $\Psi$ is not one-to-one. Let $v_{1}, v_{2} \varepsilon I_{R}\left(L_{2}\right)$, be such that $v_{1} \neq v_{2}$ and $\Psi\left(v_{1}\right)=\Psi\left(v_{2}\right)=\mu$. Since $v_{1} \neq v_{2}$ there exist $A, B \in L_{2}$ with $A \cap B=\phi$ such that 
$v_{1}(A)=1, v_{2}(A)=0, \quad v_{1}(B)=0$, and $v_{2}(B)=1$. Since $L_{1}$ partiy separates $L_{2}$, there exists $C \in \mathbf{L}_{1}$ such that A C ․ B'. Therefore, $v_{1}(C)=1$ so $\mu(C)=1$. But $\mu(C)=v_{2}(C)=0$, a contradiction.

We now present a characterization of normal lattices if the lattice is a $\delta$-lattice.

THEOREM 3.2. Suppose $L$ is a $\delta$-lattice. Then $L$ is normal if and only if the following two conditions are satisfied:

a) If $L \varepsilon L$ and if $L=\bigcap_{n=1}^{+\infty} L_{n}, L_{n} \in L$ then $L \varepsilon Z(L)$.

b) Z(L) partly separates L.

PROOF. 1. If $L$ is normal then since $L$ is $\delta$, a) follows from Alexandroff [8] (cf. Lemma 7 p. 320.) A1so, since $Z(L)$ separates $L, Z$ (L) partly separates L. 2.Z(L) is normal and since $Z(L)$ partly separates $L, Z$ (L) separates $L$. Therefore, $L$ is normal.

COROLlARY 3.1. A topological space is normal if and only if the following two condtions are satisfied:

a) Every closed set which is a $G_{\delta}$-set is a zero set.

b) For every closed set $F$ and every open set $G$ such that $F \subset G$, there exists a zero set $Z$ such that $F, Z<G$.

PROOF. Follows immediately from Theorem 3.2, letting $\mathbf{L}=\mathbf{F}$.

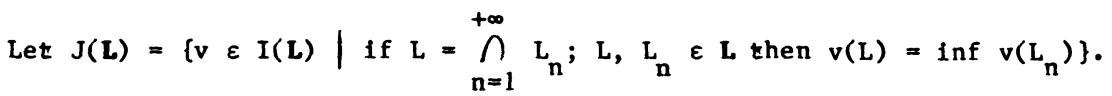

We now state a consequence of a normal lattice being complement generated which genera11zes a we11-known theorem.

THEOREM 3.3. If $L$ is normal and complement generated then $\mu \in J(L)$ implies $\mu \in I_{R}(\mathbf{L})$.

PROOF. Suppose $\mathbf{L}$ is normal and complement generated. Let $\mu \in J(L)$ and let

$L \in$ L. Then $L=\bigcap_{n}^{+\infty} L_{n}$ ' where we may assume that $L_{n}+, L_{n} \varepsilon L$ for all n. Since $L$ is normal there exist $A_{n}^{n=1}, B_{n}^{n} \varepsilon L$ such that $L \subset A_{n}^{\prime} \subset B_{n} \subset L_{n}^{\prime}$ for all $n$. Since

$\mu \varepsilon J(L), \mu(L)=\operatorname{lnf} \mu\left(B_{n}\right)=\inf \left(A_{n}{ }^{\prime}\right)$. Therefore $\mu \varepsilon I_{R}(L)$.

COROLLARY 3.2. If a $\mathrm{Z}$-filter is prime and closed under countable intersections then this Z-filter is a Z-ultrafilter with the countable intersection property.

PROOF. Follows immediately from the above theorem and the well-known correspondence between filters and measures.

The following lemma will be useful in our development of strongly normal 1attices:

LEMMA 3.2. Let $\mu_{1}, \mu_{2} \varepsilon I(L)$. If $\mu_{1} \Lambda \mu_{2} \varepsilon I(L)$ then either $\mu_{1}<\mu_{2}$ (L) or $\mu_{2}<\mu_{1}(\mathbf{L})$.

PROOF. Suppose $\mu_{1} \leqslant \mu_{2}(\mathrm{~L})$ and $\mu_{2} \leqslant \mu_{1}(\mathrm{~L})$. Then there exist A, B $\varepsilon$ L such that 
$\mu_{1}(A)=\mu_{2}(B)=1$ and $\mu_{1}(B)=\mu_{2}(A)=0$. Therefore $\mu_{1} \wedge \mu_{2}(A \cup B)=1$. Bu: $\mu_{1} \wedge \mu_{2}(A)=\mu_{1} \Lambda \mu_{2}(B)=0$ and hence $\mu_{1} \Lambda \mu_{2} \notin I(\mathbf{L})$.

Let $\tilde{I}(\mathbf{L})=\{\pi \varepsilon \Pi(\mathbf{L}) \mid$ if $A \cup B=X ; A, B \varepsilon \mathbf{l}$ then $\pi(A)=1$ or $\pi(B)=1\}$.

THEOREM 3.4. Suppose $I(L)=\tilde{I}(\mathbf{L})$. Then each of the following is $t$ rue:

a) If $\mu<\pi(\mathbf{L}), \mu \varepsilon I(\mathbf{L}), \pi \in \Pi(\mathbf{L})$ then $\pi \in I(\mathbf{L})$.

b) If $\mu<\mu_{1}(\mathbf{L})$ and $\mu \leqslant \mu_{2}(\mathbf{L}), \mu, \mu_{1}, \mu_{2} \varepsilon$ I(L) then $\mu_{1} \leqslant \mu_{2}(\mathbf{L})$ or $\mu_{2} \leqslant \mu_{1}(\mathbf{L})$.

C) L is normal.

Note: We say that $L$ is strongly normal if L satisfies $b$ ).

PROOF. a) Suppose $\mu<\pi(\mathbf{L})$ where $\mu \varepsilon I(\mathbf{L})$ and $\pi \varepsilon \Pi(\mathbf{L})$. Let $A \quad B=X$, $A, B \varepsilon$ L. Then $\pi(A \cup B)=1$ and $\mu(A \cup B)=1$. Therefore $\mu(A)=1$ or $\mu(B)=1$ and hence $\pi(A)=1$ or $\pi(B)=1$. It follows that $\pi \varepsilon \tilde{I}(\mathbf{L})=I(\mathbf{L})$. b) Let $\mu<\mu_{1}(\mathbf{L})$ and $\mu<\mu_{2}(\mathbf{L})$, where $\mu, \mu_{1}, \mu_{2} \varepsilon I(\mathbf{L})$. Now $\mu_{1} \Lambda \mu_{2} \varepsilon \tilde{I}(\mathbf{L})=I(\mathbf{L})$. Applying Lemma 3.2 completes the proof. c) Follows immediately from b) and Theorem 3.1 noting that if $\mathrm{v}_{1} \leqslant \mathrm{v}_{2}(\mathrm{~L})$, where $\mathrm{v}_{1}, \mathrm{v}_{2} \in \mathrm{I}_{\mathrm{R}}(\mathrm{L})$, then $\mathrm{v}_{1}=\mathrm{v}_{2}$.

REMARK 3.1. If $X$ is a topological space and $L=Z$, then $I(L)=\tilde{I}(L)$. Therefore, the following well-known corollary is immediate.

COROLLARY 3.3. a) A Z-filter $F$ is prime if and only if $F$ contains a prime Zfilter. b) $\mathrm{Z}$ is strongly normal.

We now show that $I(L)=\tilde{I}(L)$ if $L$ is an algebra.

THEOREM 3.5. If $\mathbf{L}=\mathbf{L}^{\prime}$ then $I(\mathbf{L})=\tilde{I}(\mathbf{L})$.

PROOF. Let $\pi \in \tilde{I}(L)$ and $F$ the corresponding filter. For each $A \varepsilon L$ either $A$ or $A^{\prime} \varepsilon F$ since $L$ is an algebra. Let $H$ be an L-filter containing $F$. Suppose $L \varepsilon H$ and $L \& F$. Then $L^{\prime} \notin \mathbf{H}$. But $L^{\prime} \varepsilon \mathbf{F C H}$ and this is a contradiction. Threfore, $F$ is an L-ultrafilter and hence a prime filter.

REMARK 3.2. If $L_{1} \subset L_{2}$ and $L_{1}^{\prime}$ separates $L_{2}^{\prime}$ then it can be shown that any $\pi_{1} \varepsilon \tilde{I}\left(\mathbf{L}_{1}\right)$ can be extended to $\pi_{2} \varepsilon \tilde{I}\left(\mathbf{L}_{2}\right)$. Such is the case for example, if $L_{1}$ equals the lattice generated by the regular open sets and $L_{2}$ equals the open sets of a topological space $X$.

\section{ON $W_{\sigma}(\mathbf{L})$ AND REgULAR LATTICES.}

$I_{R}(\mathbf{L})$ with the Wallman topology has been investigated by many writers. In this section we investigate measure conditions on $I_{R}{ }^{\sigma}(L)$ which are equivalent to $W_{\sigma}(L)$, or possibly $\tau W_{\sigma}(L)$, being $T_{2}$, regular or prime complete. We begin with a result concerning $I_{R}(\mathbf{L})$ which is not generally known.

THEOREM 4.1. Suppose $L$ is disjunctive. Then the following are equivalent:

1. W(L) is normal.

2. $W(L)$ is regular.

3. $W(\mathbf{L})$ is $T_{2}$.

PROOF. (1. implies 2.) $W(L)$ is normal and disjunctive and therefore regular. 
(2. implies 3.) $W(\mathbf{L})$ is regular and $T_{1}$ and therefore $T_{2}$ (3. implies 1. ) If $W(L)$ is $\mathrm{T}_{2}$ then $\mathrm{L}$ is normal. Therefore $W(L)$ is normal.

Suppose $L$ is disjunctive. If $\mu \varepsilon I(L)$, let $\tilde{\mu}\left(W_{\sigma}(A)\right)=\mu(A)$ for all A $\varepsilon$ A (L). Then $\tilde{\mu} \varepsilon I\left(W_{\sigma}(L)\right)$. Conversely, for any $\tilde{\mu} \varepsilon I\left(W_{\sigma}(L)\right)$ we generate a $\mu \varepsilon I(\mathbf{L})$.

We now present a measure theoretic characterization with respect to $I_{R}{ }^{\sigma}(L)$ being $\mathrm{T}_{2}$. We begin with a lemma.

LEMMA 4.1. Assume $L$ is disjunctive. Let $v \in I(L)$. If $\lambda \in I_{R}{ }^{\sigma}(L)$ then $v \in S(\tilde{\mu})$ if and only if $\mu<\mathrm{v}(\mathrm{L})$.

PROOF. Suppose $v \varepsilon S(\tilde{\mu})$. Then $\mu(L)=1+\tilde{\mu}\left(W_{\sigma}(L)\right)=1+v \varepsilon W_{\sigma}(L)+v(L)=1$, L $\varepsilon$ L.

Conversely, suppose $\mu<v(L) . \quad \tilde{u}\left(W_{\sigma}(L)\right)=1+\mu(L)=1+v(L)=1$. $v \varepsilon W_{\sigma}(L)+v \varepsilon S(\tilde{\mu}), L \varepsilon L$.

THEOREM 4.2. Suppose $L$ is disjunctive. Then $I_{R}{ }^{\sigma}(L)$ with the Wallman topology is $\mathrm{T}_{2}$ if and only if for all $\mu \in \mathrm{I}(\mathrm{L}), \quad$ if $\mu<\mathrm{v}_{1}$ (L) and $\mu<v_{2}(\mathrm{~L})$, where $v_{1}, v_{2} \varepsilon I_{R}^{\sigma}(L)$, then $v_{1}=v_{2}$.

PROOF. Suppose $I_{R}{ }^{\sigma}(L)$ is $T_{2}$. Then $\tau W_{\sigma}(L)$ is $T_{2}$ and hence $W_{\sigma}(L)$ is $T_{2}$. Let $\mu$, ' $v_{1}$ and $v_{2}$ be as above. By Lemma $4.1, v_{1}, v_{2} \varepsilon S(\tilde{\mu})$. Since $W_{g}(L)$ is $T_{2}, v_{1}=v_{2}$.

Converse1y, assume $\mu<v_{1}(L)$ and $\mu<v_{2}(L)$, where $v_{1}, v_{2} \varepsilon I_{R}{ }^{\circ}(L)$, implies that $v_{1}=v_{2}$. Suppose $S(\tilde{\mu}) \neq \phi$. If $v_{1}, v_{2} \varepsilon S(\tilde{\mu})$ then $\mu<v_{1}(L)$ and $\mu<v_{2}(L)$. Therefore $v_{1}=v_{2}$. Thus $W_{\sigma}(L)$ and hence $\tau W_{\sigma}(L)$ is $T_{2}$.

Consider the following condition which we call condition A: For all $\mu_{1}, \mu_{2} \varepsilon I(L)$ such that $\mu_{1}<\mu_{2}(L)$, if $v \varepsilon I_{R}{ }^{\sigma}(L)$ and $\mu_{1}<v(L)$ then $\mu_{2}<v(\mathbf{L})$.

We now show that condition $A$ is equivalent to $W_{\sigma}(L)$ being regular if $L$ is disjunctive.

THEOREM 4.3 Suppose $L$ is disjunctive. Then $W_{\sigma}(L)$ is regular if and only if condition $A$ is true.

PROOF. Let $\mu \varepsilon I(L)$ and $\tilde{\mu}\left(W_{\sigma}(A)\right)=\mu(A), A \varepsilon A(L)$. Assume $W_{\sigma}(L)$ is regular. If $\mu_{1}<\mu_{2}(L)$ where $\mu_{1}, \mu_{2} \in I(L)$ then $\tilde{\mu}_{1}<\tilde{\mu}_{2}(W(L))$. Therefore $S\left(\tilde{\mu}_{1}\right)=S\left(\tilde{\mu_{2}}\right)$. The condition follows by applying Lemma 4.1. Conversely, one may easily show by applying Lemma 4.1 that if condition A holds then $\tilde{\mu}_{1}<\tilde{\mu}_{2}(W(L))$ implies that $S\left(\tilde{\mu}_{1}\right)=S\left(\tilde{\mu}_{2}\right)$ and hence $W_{\sigma}(L)$ is regular.

We observe that condition A implies that if $\mu \varepsilon I(L)$ then there exists at most one $v \in I_{R}{ }^{\sigma}(\mathrm{L})$ such that $\mu<v(\mathrm{~L})$. Therefore, applying Theorems 4.2 and 4.3 we have: COROLlaRY 4.1. Suppose $L$ is disjunctive. If $W_{\sigma}(L)$ is regular then $W_{\sigma}(L)$ is $T_{2}$.

The following two theorems give conditions which guarantee that condition $A$ is true.

THEOREM 4.4. If $I(L)=\tilde{I}(\mathbf{L})$ then condition $A$ is true.

PROOF. Since $I(L)=\tilde{I}(L), L$ is strongly normal. Therefore, if $\mu_{1}<\mu_{2}(L)$, $\mu_{1}, \mu_{2} \varepsilon I(\mathbf{L})$, and if $\mu_{1}<v(\mathbf{L}), \nu \varepsilon I_{R}^{\sigma}(\mathbf{L})$, then either $\mu_{2}<v(\mathbf{L})$ or $\nu<\mu_{2}(\mathbf{L})$. Since $\nu \in I_{R}^{\sigma}(L), \mu_{2}<\nu(L)$.

COROLLARY 4.2. If $I$ is disjunctive and $I(L)=\tilde{I}(L)$ then $I_{R}^{\sigma}(L)$, with the Wallman topology, is regular. 
PROOF. If $I(L)=\tilde{I}(L)$ then condition $A$ is true. Therefore, $W_{\sigma}(L)$ is regular and hence $\tau W_{j}(\mathbf{L})$ is regular.

THEOREM 4.5. If $\mathbf{L}$ is regular and replete then $L$ satisfies condition A.

PROOF. Suppose $\mu_{1} \leqslant \mu_{2}(\mathbf{L}), \mu_{1}, \mu_{2} \in I(\mathbf{L})$ and $\mu_{1}<v(\mathbf{L}), v \varepsilon I_{R}^{\sigma}(\mathbf{L})$. Since $\mathbf{L}$ is regular, $S\left(\mu_{1}\right)=S\left(\mu_{2}\right)=S(\nu)$ and since $L$ is replete, $S(\nu) \neq \phi$. Let $x \in S(\nu)$. Then $\nu<\mu_{x}(L)$ where $\mu_{x}$ is the measure concentrated at $x$. But $\mu_{x} \varepsilon I_{R}{ }^{\sigma}(L)$ and therefore, $\nu=\mu_{x}$. Since $x \in\left(\mu_{2}\right), \mu_{2} \leqslant v(L)$.

our next goal is to find a condition which guarantees that condition $A$ on $L_{1}$ implies condition $A$ on $L_{2}$ where $L_{1} \subset L_{2}$. We begin with a lemma.

LEMMA 4.2. Suppose $\mathbf{L}_{1} \subset \mathbf{L}_{2}$ and $\mathbf{L}_{1}$ separates $\mathbf{L}_{2}$. Let $\mu \varepsilon I(\mathbf{L}), v \varepsilon I_{R}(\mathbf{L})$, and let $\rho$ and $\tau$ respectively be extensions of $\mu$ and $\nu$ to $\left(L_{2}\right)$ where $\tau \varepsilon I_{R}\left(\mathbf{L}_{2}\right)$. If $\mu<\nu\left(\mathbf{L}_{1}\right)$ then $\rho<\tau\left(\mathbf{L}_{2}\right)$.

PROOF. Suppose $\tau\left(A_{2}\right)=0, A_{2} \varepsilon L_{2}$ since $\tau \in I_{R}\left(L_{2}\right)$, there exists $B_{2} \varepsilon L_{2}$ such that $A_{2} \subset B_{2}$ and $\tau\left(B_{2}\right)=0$. Since $L_{1}$ separates $L_{2}$, there extst $A_{1}, B_{1} \varepsilon L_{1}$ such that $B_{2} C A_{1}, A_{2} \subset B_{1}$ and $A_{1} \cap B_{1}=\phi$. It follows that $\rho\left(A_{2}\right)=0$. .

THEOREM 4.6. Suppose $L_{1} \subset L_{2}$ and $L_{1}$ separates $L_{2}$. If condition $A$ holds on $L_{1}$ then condition $A$ holds on $L_{2}$.

PROOF. Let $v_{1}<v_{2}\left(\mathbf{L}_{2}\right)$ where $v_{1}, v_{2} \varepsilon I\left(\mathbf{L}_{2}\right)$ and $v_{1}<v\left(\mathbf{L}_{2}\right)$ where $v \varepsilon I_{R}{ }^{\sigma}\left(\mathbf{L}_{2}\right)$. Let $\mu_{1}, \mu_{2}$ and $\mu$ respectively be the restrictions of $v_{1}, v_{2}$ and $v$ to $A\left(L_{1}\right)$. Then by condition $A$ on $L_{1}, \mu_{2} \leqslant \mu\left(L_{1}\right)$. The proof now follows from Lemma 4.2.

Clearly, if $\mathbf{L}$ is regular then $\tau \mathrm{L}$ is regular. The following two theorems consider conditions which guarantee the converse.

THEOREM 4.7. Assume $\tau$. is regular. Let $\mu_{1}<v_{1}(L), \mu_{1}, v_{1} \varepsilon I(L)$. If there exist $\mu_{2}, v_{2} \varepsilon I(\tau L)$ such that $\mu_{2}$ and $v_{2}$ restricted equal $\mu_{1}$ and $v_{1}$ respectively and if $\mu_{2}<v_{2}(\tau L)$ then $L$ is regular.

PROOF. Clearly if $\mu_{1}<v_{1}(L)$ then $S\left(\mu_{1}\right)=S\left(\mu_{2}\right)=S\left(v_{2}\right)=S\left(v_{1}\right)$ under the above hypotheses.

THEOREM 4.8. Assume $L$ is normal and $L$ semiseparates $\tau L$. Then if $\tau L$ is regular, $L$ is regular.

PROOF. Let $\mu_{1}, \mu_{2}$ and $v_{1}$ be as in Theorem 4.7. Let, $\rho \in I_{R}(\mathbf{L})$ and $v_{1}<\rho(L)$. Similarly, let $v \in I_{R}(\tau L)$ and $\mu_{2}<v(\tau L)$. Since L semiseparates $\tau L, v$ restricted to $A(L)$ is L-regular and equals $\rho$ since $L$ is normal. Therefore, $S\left(\mu_{1}\right)=S\left(v_{1}\right)$.

We now find a measure theoretic condition which is equivalent to $W_{\sigma}(L)$ being prime complete.

THEOREM 4.9. Suppose $L$ is disjunctive. $W_{\sigma}(L)$ is prime complete if and only if for all $\mu \varepsilon I_{\sigma}(\mathbf{L})$ there exists $V \in I_{R} \sigma_{(L)}$ such that $\mu<v(L)$.

PROOF. Follows immediately from Lemma 4.1 .

COROLLARY 4.3. Suppose $L$ is disjunctive, normal and countably paracompact. Then $W_{\sigma}(L)$ is prime complete.

PROOF. Let $\mu \varepsilon I_{\sigma}(L)$ Since $L$ is normal and countably paracompact, there exists $\quad v \in I_{R}{ }^{\sigma}(L)$ such that $\mu<v(L)$. 
Finally, we give the following theorem which extends the known measure theoretic consequence of regular lattices to $M(L)$

THEOREM 4.10. Suppose $L$ is regular. Let $\mu_{1}, \mu_{2} \in M(\mathbf{L})$. If $\mu_{1}<\mu_{2}(\mathbf{L})$ and $\mu_{1}(X)=\mu_{2}(X)$ then $S\left(\mu_{1}\right)=S\left(\mu_{2}\right)$.

PROOF. Clearly $S\left(\mu_{2}\right) \subset S\left(\mu_{1}\right)$.

Let $x \in S\left(\mu_{1}\right)$. If $x \in S\left(\mu_{2}\right)$ then there exists $L \in L$ such that $\mu_{2}(L)=\mu_{2}(X)$ and $x \in L$. Since $L$ is regular there exist $A, B \in L$ such that $x^{\prime} \varepsilon A^{\prime}, L \subset B^{\prime}$ and $B^{\prime}$. A. Therefore, $\mu_{2}\left(B^{\prime}\right)=\mu_{2}(X)=\mu_{1}(X)$. Therefore, $\mu_{1}(A)=\mu_{1}(X)$ hence $x \in A$ which is a contradiction.

\section{REFERENCES}

1. GRASSI, P. On Subspaces of Replete and Measure Replete Spaces, Canad. Math. Bu11. 1 (1984) 27 .

2. HUERTA, C. Notions of Compactness on the Lattice and on the Point Set in Terms of Measure, Ann. Sc. Math., Quebec, 13(1) (1989), 49-52.

3. NOBELING, G. Grundlagen der Analytichen Topologie, Springer Verlang, Berline, 1954.

4. SZETO, M. On Maximal Measures with Respect to a Lattice, Measure Theorey and its Applications, 1980, Northern Illinois University, 277-282.

5. SzEto, M. Measure Repleteness and Mapping Preservations, Jour. Ind. Math. Soc. 43 (1979), 35-52.

6. FROLIK, Z. Prime Filters with the C.I.P., Comm. Math. Univ. Carolinae, 13(3) (1972), 553-573.

7. GRASSI, P. An Adjoint Map between Regular Measures and it Applications Jour. Ind. Math. Soc. 48 (1984), 19-31.

8. ALEXANDROFF, A.D. Additive-set Functions in Abstract Spaces, Mat. Sb., (N.S.) 8 $50(1940), 207-348$. 


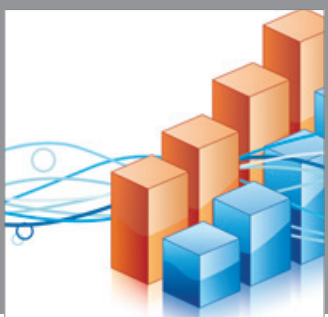

Advances in

Operations Research

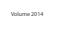

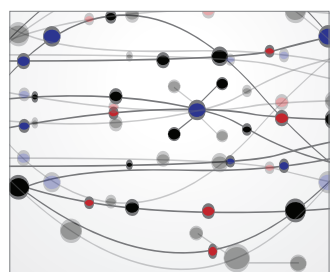

\section{The Scientific} World Journal
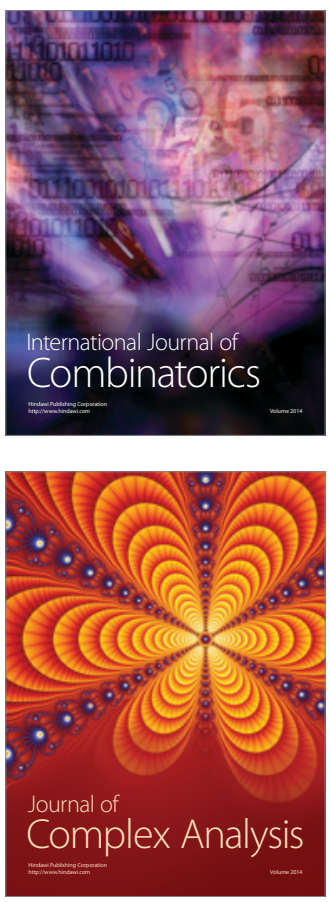

International Journal of

Mathematics and

Mathematical

Sciences
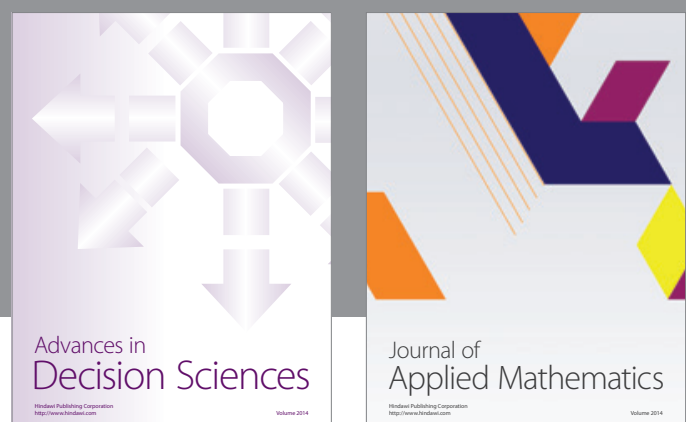

Journal of

Applied Mathematics
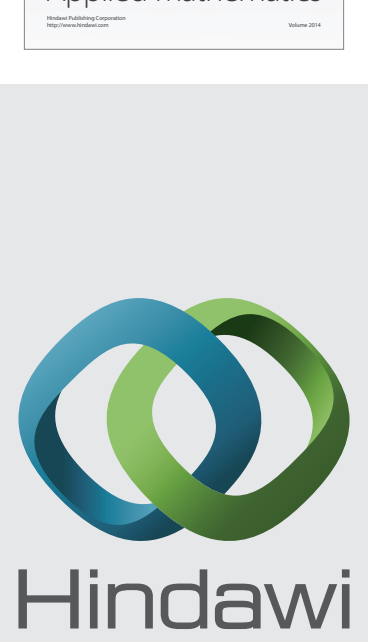

Submit your manuscripts at http://www.hindawi.com
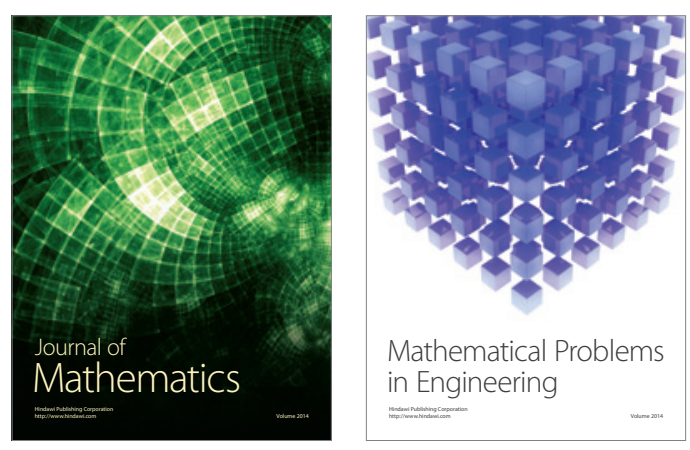

Mathematical Problems in Engineering
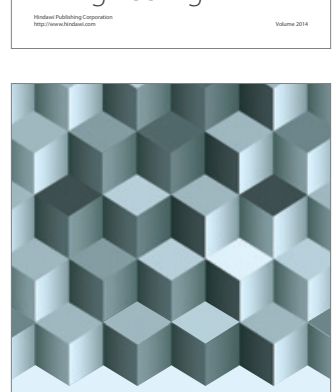

Journal of

Function Spaces
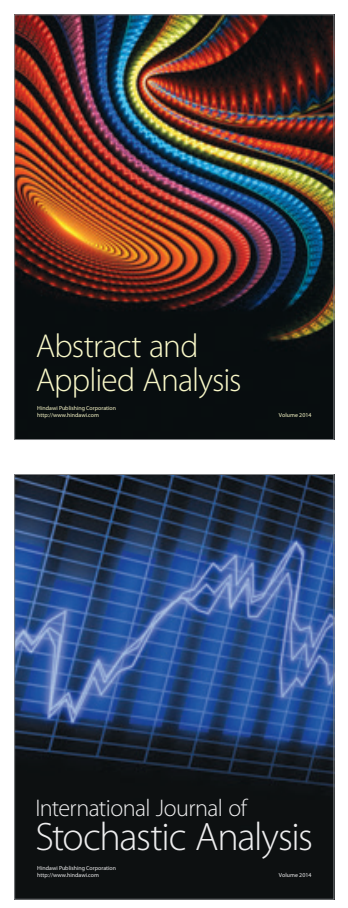

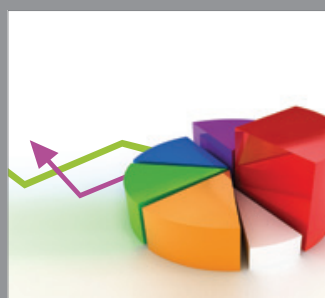

ournal of

Probability and Statistics

Promensencen
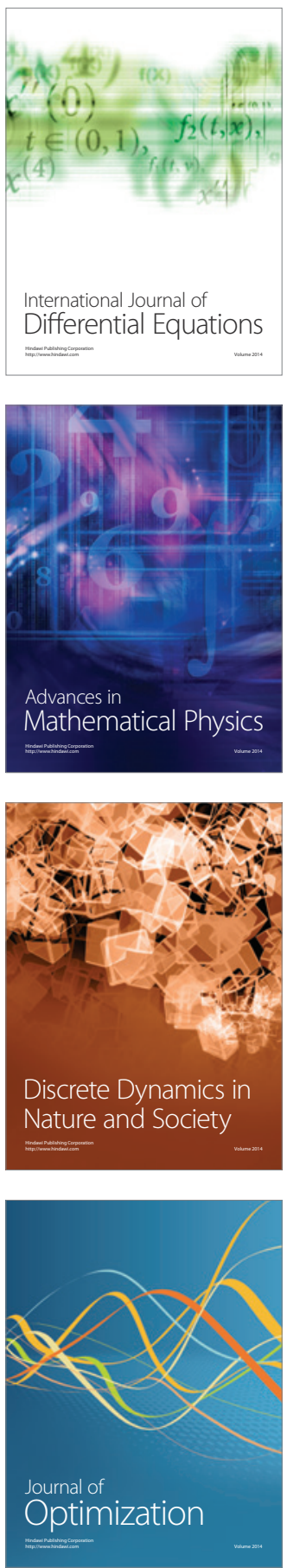